\title{
A multicolor large Stokes shift fluorogen-activating RNA aptamer with cationic chromophores
}

\author{
Christian Steinmetzger, ${ }^{[a]}$ Navaneethan Palanisamy, ${ }^{[b] \#}$ Kiran R. Gore ${ }^{[c] \S}$ and Claudia Höbartner*[a,b,c]
}

\begin{abstract}
Large Stokes shift (LSS) fluorescent proteins (FPS) exploit excited state proton transfer pathways to enable fluorescence emission from the phenolate intermediate of their internal 4-hydroxybenzylidene imidazolone (HBI) chromophore. An RNA aptamer named Chili mimics LSS FPs by inducing highly Stokesshifted emission from several new green and red $\mathrm{HBI}$ analogs that are non-fluorescent when free in solution. The ligands are bound by the RNA in their protonated phenol form and feature a cationic aromatic side chain for increased RNA affinity and reduced magnesium dependence. In combination with oxidative functionalization at the $C 2$ position of the imidazolone, this strategy yielded $D M H B O^{+}$, which binds to the Chili aptamer with a low-nanomolar $K_{D}$. Because of its highly red-shifted fluorescence emission at $592 \mathrm{~nm}$, the Chili-DMHBO ${ }^{+}$complex is an ideal fluorescence donor for Förster resonance energy transfer (FRET) to the rhodamine dye Atto 590 and will therefore find applications in FRET-based analytical RNA systems.
\end{abstract}

Fluorogen-activating RNA aptamers (FLAPs) have emerged as powerful tools for tagging and visualizing RNA in vitro and in vivo, and, among various other applications in biochemistry and synthetic biology, they are used as components of metabolite and protein sensors. ${ }^{[1]}$ These artificial functional RNAs form specific, non-covalent complexes with conditionally fluorescent chromophores that are non-emissive free in solution, but show strongly enhanced fluorescence emission in the bound state. Early examples, including the Malachite green aptamer ${ }^{[2]}$ and the sulforhodamine aptamer, ${ }^{[3]}$ are currently receiving renewed attention for further optimization and reselection, ${ }^{[4,5]}$ and new chromophore-binding aptamers, such as cyanine dye-activating Dir2s ${ }^{[6]}$ and Mango RNA aptamers ${ }^{[7,8]}$ continue to be developed. A prominent class of fluorogenic aptamers known as mimics of fluorescent proteins, named Spinach, ${ }^{[9]}$ Broccoli, ${ }^{[10]}$ and Corn, ${ }^{[11]}$ were evolved to bind analogs of the GFP chromophore 4hydroxybenzylidene imidazolone (HBI). Synthetic $\mathrm{HBI}$ derivatives are non-fluorescent in aqueous solution, but non-

\footnotetext{
[a] C. Steinmetzger, Prof. Dr. C. Höbartner Institute of Organic Chemistry, University of Würzburg Am Hubland, 97074 Würzburg (Germany) E-mail: claudia.hoebartner@uni-wuerzburg.de

[b] N. Palanisamy, Prof. Dr. C. Höbartner International Max Planck Research School Molecular Biology, University of Göttingen (Germany)

\# present address: BIOSS Center for Biological Signaling Studies, University of Freiburg (Germany)

[c] Dr. K.R. Gore, Prof. Dr. C. Höbartner Center for Nanoscale Microscopy and Molecular Physiology of the Brain (CNMPB), Göttingen, (Germany)

$\S \quad$ present address: Department of Chemistry, University of Mumbai (India)
}

Supporting information for this article is given via a link at the end of the document. covalent binding to an RNA aptamer prevents non-radiative deactivation of the excited state, thereby restoring the intrinsic fluorescence.

The majority of known fluorogenic aptamers is characterized by a small or moderate difference between their excitation and emission maxima. However, fluoromodules with large Stokes shifts display significant advantages, such as reduced reabsorption of the emitted light, ${ }^{[12]}$ convenient use as FRET donors for far-red fluorescence reporters,${ }^{[13]}$ and the ability to produce multiple emission colors by simultaneous excitation of different fluorophores at a single wavelength. ${ }^{[14]}$ Large energy differences between absorption and emission peaks can result from intramolecular charge transfer (ICT), or from inter- or intramolecular excited state proton transfer (ESPT), among other mechanisms. ${ }^{[12]}$ Fluorescent proteins that exhibit large apparent Stokes shifts (termed LSS FPs) have been engineered to exploit ESPT from the aromatic $\mathrm{OH}$ group of the $\mathrm{HBI}$ chromophore to a neighboring amino acid of the $\beta$-barrel scaffold. Prominent members of this family are the orange/red emitting proteins LSSmOrange ${ }^{[14]}$ and LSSmKate ${ }^{[15]}$ which are excited at 437 and $460 \mathrm{~nm}$ and emit at 572 and $605 \mathrm{~nm}$, respectively. Similarly large Stokes shifts and fluorescence emission in the $600 \mathrm{~nm}$ region have not been reported for fluorogenic RNA aptamers. In this study, we aim to mimic LSS green and red fluorescent proteins by capitalizing on fluorogenactivating RNA aptamers that bind the protonated form of $\mathrm{HBI}$ analogs and enable ESPT and emission from an excited deprotonated intermediate.

Spinach, Broccoli and Corn are members of the HBI-binding aptamer family that activate derivatives of 3,5-difluoro-HBI (DFHBI), which are almost completely deprotonated at physiological $\mathrm{pH} \cdot{ }^{[9]}$ Here, we capitalized on 3,5-dimethoxy-substituted $\mathrm{HBI}(\mathrm{DMHBI})$ derivatives that have a higher $\mathrm{p} K_{\mathrm{a}}$ and can be excited at the absorption maximum of the phenol form. We first examined the 13-2 aptamer, which was originally reported to activate the fluorescence of DMHBI, showing an emission at 529 $\mathrm{nm}$ upon excitation at $398 \mathrm{~nm} \cdot{ }^{\left[{ }^{9]}\right.}$ Based on the analysis of the predicted secondary structure, ${ }^{[16]}$ we introduced an extra-stable UUCG tetraloop into 13-2min, with the intention to stabilize an apical hairpin and to assist folding of the ligand binding pocket. This modified 52-nt RNA aptamer (Figure 1) showed approx. 2fold enhanced fluorescence of bound DMHBI compared to the original 60-nt RNA, and the fraction of RNA that folded into a ligand binding-competent conformation was significantly enhanced (Figure S1). The 52-nt RNA resulting from the optimization of 13-2min was used for all further experiments discussed in this manuscript, and - in line with the "vegetable nomenclature" - was named Chili because of its special properties to activate green, yellow and red chromophores. 
a)<smiles>COc1cc(/C=C2\N=C(C)N(C)C2=O)cc(OC)c1O</smiles>

DMHBI<smiles>COc1cc(/C=C2\N=C(/C=C/c3c[nH]cn3)N(C)C2=O)cc(OC)c1O</smiles>
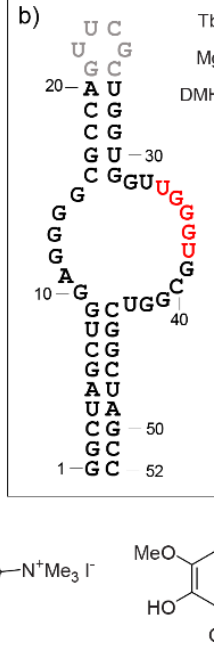

$\mathrm{Tb}^{3+} 10 \mathrm{mM} \quad 0.5 \mathrm{mM}$ $\mathrm{Mg}^{2+} \overline{++-} \overline{++-F}$

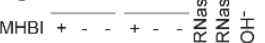

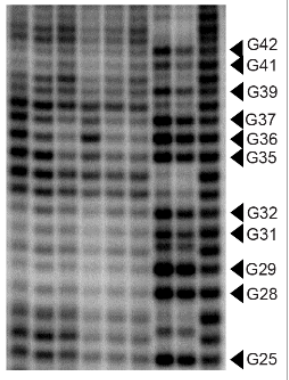

OMe

$\mathrm{DMHBO}^{+}$
$\mathrm{DMHBI}^{+}$

Figure 1. a) Structures and names of the $\mathrm{HBI}$ derivatives investigated in this work. b) Sequence of 52-nt Chili RNA and gel image of $\mathrm{Tb}^{3+}$-footprinting, comparing unfolded and folded RNA in the absence and presence of DMHBI. Full gel image in Figure S3. The flexible loop region is indicated in red.

The high guanine content of the Chili RNA sequence suggests that it contains guanine quartets in the ligand binding site. This hypothesis is supported by the finding that DMHBI fluorescence is strongly dependent on the $\mathrm{K}^{+}$concentration with an apparent $K_{\mathrm{D}}$ of $12 \mathrm{mM}$ and a Hill coefficient of 2.5 (Figure S2), suggesting the involvement of a two-tiered $G$ quadruplex. ${ }^{[17,18]}$ This feature is reminiscent of other $\mathrm{HBI}$-binding aptamers, including Spinach and Corn, for which the crystal structures show highly unusual quadruplex topologies, ${ }^{[18]}$ as well as Mango aptamers, which also contain quadruplexes as ligand-binding structural elements. ${ }^{[19]}$ Despite the overall similarity of the $2 \mathrm{D}$ secondary structure schemes of Chili and Spinach/Broccoli (Figure S1), their sequences are significantly different. In the absence of a 3D structural model, it remains unknown which guanines of Chili are involved in putative $\mathrm{G}$ quartets. However, it is interesting to note that, compared to Spinach, Chili has additional nucleotides that are expected to form larger loops extruding from a putative quadruplex core. This is supported by structural probing data that clearly identify the region of nt 34-38 in the folded and ligand-bound state as more susceptible to backbone hydrolysis compared to the unfolded state, i.e., in the absence of ligand and $\mathrm{Mg}^{2+}$ (Figure $1 \mathrm{~b}$ and Figure S3).

The presence of the flexible bulge in Chili suggested an opportunity to modulate the fluorescence properties of the RNAchromophore complexes by ligand engineering. Candidate sites for modification are the positions $\mathrm{N} 1$ and $\mathrm{C} 2$ of the imidazolone, similar to variations observed in fluorescent proteins, originating from the primary protein sequences. In analogy, the DFHBI ligand was earlier modified at these two sites, resulting in DFHBI-1T as a brighter Spinach ligand ${ }^{[20]}$ and DFHO as the ligand responsible for orange emission in complex with the Corn aptamer. $^{[11]}$

Here, we designed and synthesized three new DMHBI ligands (Figure 1) and investigated their fluorescence properties in complex with the Chili RNA. The $\mathrm{DMHBI}^{+}$derivative carries a trimethylammonium-substituted phenyl ring at N3, which may provide additional stacking interactions with nucleobases, and the cationic substituent may assist electrostatic interactions with the negatively charged RNA backbone. These features should enforce a rigid fluorophore conformation and stabilize the position of the fluorophore at the binding site. The other two new $\mathrm{DMHBI}$ analogs were extended at the $\mathrm{C} 2$ position: $\mathrm{DMHBI}-\mathrm{Imi}$ mimics
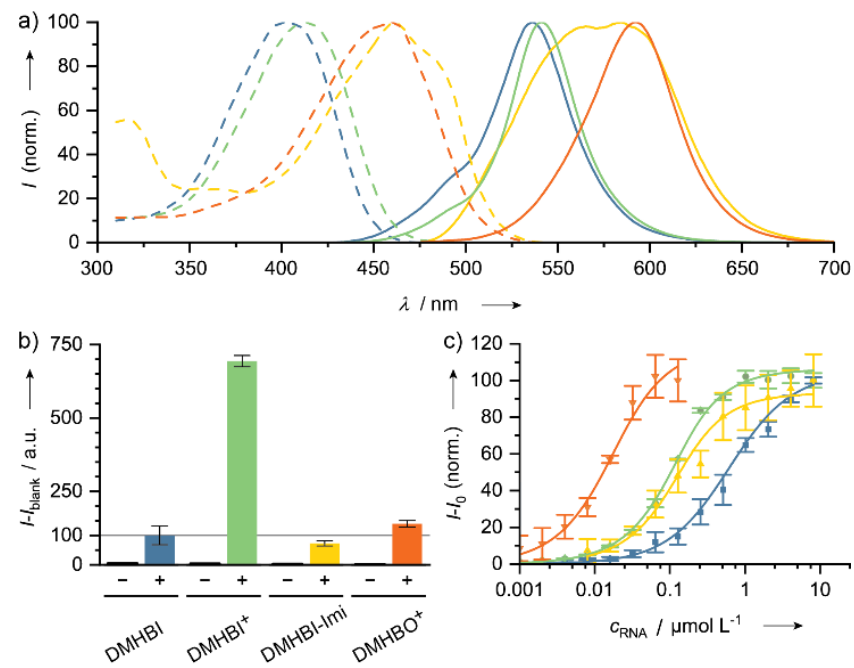

Figure 2. a) Normalized excitation and emission spectra (dashed and solid lines, resp.) of DMHBI (blue), $\mathrm{DMHBI}^{+}$(green), DMHBI-Imi (yellow), and $\mathrm{DMHBO}^{+}$(orange) bound to Chili RNA. b) Integrated fluorescence intensity of each dye in the presence (+) or absence (-) of the Chili RNA at pH 7.5. c) Titration with Chili RNA reveals higher affinity for all three new chromophores compared to $\mathrm{DMHBI}\left(K_{\mathrm{D}}\right.$ listed in Table 1 , experimental details given in supporting information).

the chromophore of the protein Kaede, which contains a histidine-derived arylvinyl substituent. ${ }^{[21]}$ Other red fluorescent proteins, such as DsRed and its relatives, carry acylimine substituents instead of arylvinyl groups at $\mathrm{C} 2 .^{[22]}$ In combination with the trimethylammoniumphenyl side chain described above, the oxime in $\mathrm{DMHBO}^{+}$was chosen as a hydrolytically stable acylimine mimic.

The three new chromophores were synthesized starting from 4-hydroxy-3,5-dimethoxybenzaldehyde (Scheme S1). $\mathrm{DMHBI}^{+}$ was obtained in three steps, starting with the condensation with 4-(dimethylamino)aniline. The imine was incorporated into the $\mathrm{HBI}$ scaffold in a 1,3-dipolar cycloaddition reaction with an imidate ylide, which was prepared from ethyl acetimidate and methyl glycinate. ${ }^{[23]}$ The aromatic dimethylamino group was alkylated with methyl iodide to obtain $\mathrm{DMHBI}^{+}$. To generate $\mathrm{DMHBO}^{+}$, the cycloaddition product was oxidized with selenium dioxide and the resulting aldehyde was reacted with hydroxylamine, followed by alkylation with methyl iodide. The Kaede analog DMHBI-Imi was generated from DMHBI via a scandium 
triflate-catalyzed Aldol condensation with imidazole-4-carboxaldehyde. ${ }^{[24]}$

The fluorescence properties of the four dyes and their binding and activation by Chili were investigated. Chili-DMHBI and Chili-DMHBI ${ }^{+}$showed excitation maxima near $400 \mathrm{~nm}$ and emission maxima near $540 \mathrm{~nm}$ (Figure 2a), demonstrating that the $\mathrm{Me}_{3} \mathrm{~N}^{+} \mathrm{Ph}$ substituent at $\mathrm{N} 3$ has little influence on the conjugated $\pi$-electron system of DMHBI. However, a more than sevenfold increase in fluorescence enhancement was observed compared to the parent DMHBI (Figure 2b), suggesting that the cationic side chain prevents non-radiative deactivation of the excited state more effectively. The other two chromophores were expected to yield red-shifted fluorescence emission. Indeed, Chili-DMHBO ${ }^{+}$showed bathochromic shifts of the excitation (455 nm) and emission maxima (592 nm), exceeding other RFP mimics like the Corn $(545 \mathrm{~nm})$ and Red Broccoli (582 $\mathrm{nm})$ systems. $^{[11]}$ Excitation of Chili-DMHBI-Imi at $463 \mathrm{~nm}$ resulted in a bimodal emission profile with maxima at 545 and $594 \mathrm{~nm}$. The fluorescence intensities of Chili-DMHBO ${ }^{+}$and Chili-DMHBI-Imi were comparable within $20 \%$ to the ChiliDMHBI complex, which is also reflected in their comparable fluorescence quantum yields (Table 1). Importantly, none of the DMHBI derivatives showed unspecific fluorescence activation, as demonstrated by control experiments with E.coli tRNA, other non-cognate RNA aptamers (including Spinach), and DNA quadruplexes (Figures S4, S5).

Binding affinity and magnesium dependence are of crucial importance for the application of fluorogenic RNA aptamers. In the absence of $\mathrm{Mg}^{2+}$, the fluorescence intensity of the Chili complexes with DMHBI and DMHBI-Imi was reduced to approx. $50 \%$ of the value obtained with $5 \mathrm{mM} \mathrm{Mg}^{2+}$. In contrast, the cationic ligands $\mathrm{DMHBI}^{+}$and $\mathrm{DMHBO}^{+}$retained approx. $80 \%$ of their maximum intensity even in the absence of $\mathrm{Mg}^{2+}$ (Figure S6), suggesting that the positively charged side chain partially compensated for the reduced availability of divalent metal ions. Other recently reported systems, in which arylvinyl-substituted $\mathrm{HBI}$ analogs were shown to be activated by parallel-stranded DNA quadruplexes, required $50 \mathrm{mM} \mathrm{Mg}^{2+}$. 25$]$

The dissociation constants of the new FLAP complexes were determined by titrating each fluorophore with increasing amounts of RNA (Figure 2c, Table 1). We found a $K_{D}$ of $570 \mathrm{nM}$ for DMHBI binding to Chili RNA, similar to the original 13-2 aptamer. ${ }^{[9]}$ For the brighter Chili-DMHBI ${ }^{+}$complex an almost $10-$ fold stronger binding was observed with a $K_{\mathrm{D}}$ of $63 \mathrm{nM}$. The DMHBI-Imi chromophore was bound with similar affinity $\left(K_{\mathrm{D}}\right.$ of $71 \mathrm{nM})$, suggesting that the bulky imidazolylvinyl substituent at C2 was also well-accommodated. Interestingly, the cationic $\mathrm{DMHBO}^{+}$chromophore displayed the tightest binding with a $K_{\mathrm{D}}$ of $12 \mathrm{nM}$, suggesting synergistic effects of the C2 and N3 substituents. Such low-nanomolar affinities have been observed for the Mango-TO1-biotin fluoromodules, ${ }^{[7,8]}$ but could not be reached by previous iterations of $\mathrm{HBI}$-binding aptamers. The increase in binding affinity and fluorescence enhancement by the $\mathrm{Me}_{3} \mathrm{~N}^{+} \mathrm{Ph}$ substituent is specific for DMHBI derivatives in complex with Chili. Analogous modification of DFHBI and DFHO (Scheme S2) completely eliminated binding and fluorescence activation by Spinach and Broccoli (Figure S5).
In consequence of this distinctive performance, the properties of $\mathrm{DMHBO}^{+}$and its complex with Chili were further investigated. The $\mathrm{p} K_{\mathrm{a}}$ of the free chromophore was determined as 6.9 (Figure 3a), which results in approx. $80 \%$ deprotonation at $\mathrm{pH}$ 7.5. and the appearance of the phenol (at $436 \mathrm{~nm}$ ) and phenolate (at $547 \mathrm{~nm}$ ) absorbance bands. Interestingly, the RNA-bound chromophore exhibited a single absorption band with a maximum at $456 \mathrm{~nm}$ (Figure 3b, S9). This suggests that the Chili RNA exclusively binds the $\mathrm{DMHBO}^{+}$chromophore in its protonated state. The bathochromic shift of $20 \mathrm{~nm}$ compared to the free phenol likely originates from a combination of $\pi$-stacking and electrostatic interactions. ${ }^{[26]}$ Excitation of the protonated chromophore-RNA complex is followed by fast deprotonation, which results in the large apparent Stokes shift of $136 \mathrm{~nm}$. These results suggest that a functional group of the RNA near the phenol acts as a proton shuttle. The identity of this functional group on a nucleobase or in the ribose-phosphate backbone remains to be identified.
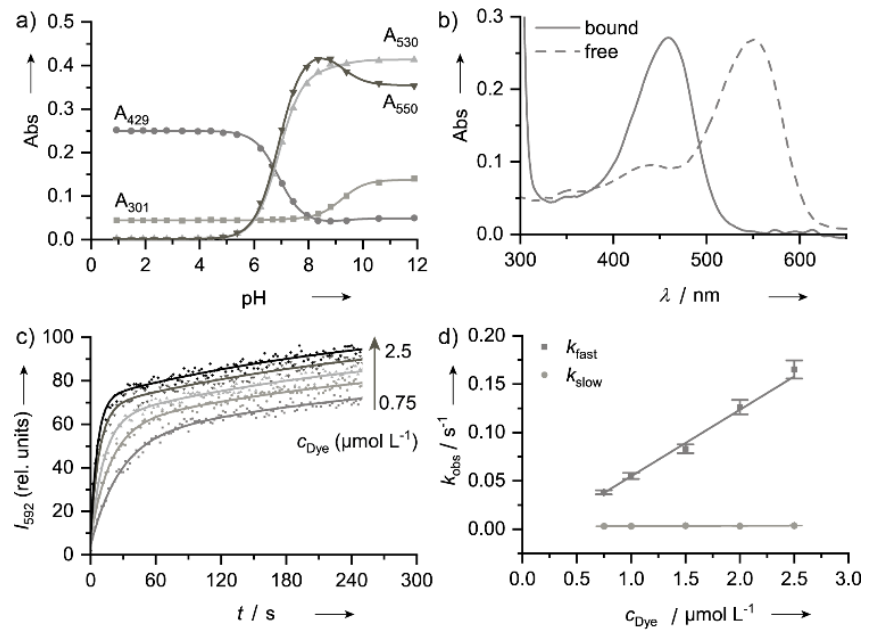

Figure 3. a) Absorbance vs $\mathrm{pH}$ titration of $\mathrm{DMHBO}^{+}$gives a $\mathrm{p} K_{\mathrm{a}}$ of 6.9 . b) The UV-VIS spectra of $\mathrm{DMHBO}^{+}$in aqueous buffer at $\mathrm{pH} 7.5$ (dashed line) and bound to the Chili aptamer (solid line). c) Time course of the fluorescence emission at $592 \mathrm{~nm}$ after mixing Chili and $\mathrm{DMHBO}^{+}$under pseudo-first order conditions follows a biexponential model. d) The apparent rate constants from c) plotted versus $\mathrm{DMHBO}^{+}$concentration.

Next, we studied the kinetics of $\mathrm{DMHBO}^{+}$activation by the Chili aptamer. The time course of fluorescence emission at $592 \mathrm{~nm}$ after mixing Chili and $\mathrm{DMHBO}^{+}$under pseudo-first order reaction conditions followed a biexponential model with two apparent rate constants (Figure 3c, d), of which only one was linearly dependent on the dye concentration. This can be interpreted as a fast binding of the chromophore to a partially preorganized binding site, followed by a slow reorganization of peripheral residues. Prolonged incubation of the RNA with $\mathrm{K}^{+}$- and $\mathrm{Mg}^{2+}$ containing buffer prior to the addition of $\mathrm{DMHBO}^{+}$had no influence on the observed association kinetics (Figure S7). Therefore, folding of the putative quadruplex is not rate-limiting and the slow refolding of the binding site likely involves loop nucleotides. Overall, the Chili LSS FLAP shows a much faster 
fluorescence turn-on compared to the slow maturation times of LSS red fluorescent proteins, which are in the range of hours. ${ }^{[14]}$

The tight binding and strong fluorescence enhancement of Chili-DMHBO ${ }^{+}$can be used to visualize RNA in native and denaturing polyacrylamide gels (Figure S13, S15). Moreover, the Chili-DMHBO ${ }^{+}$complex serves as a fluorescence donor for FRET-based analytical systems, as demonstrated with an Atto 590-labeled Chili RNA aptamer (Figure 4) that carries the

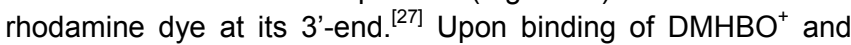
excitation at $456 \mathrm{~nm}$, fluorescence emission from Atto 590 was observed, which can be rationalized by the strong overlap of the $\mathrm{DMHBO}^{+}$emission and Atto 590 absorption bands (Figure S14). The large Stokes shift of Chili-DMHBO ${ }^{+}$rules out direct excitation of the acceptor, as shown in the absence of $\mathrm{DMHBO}^{+}$ (Figure S14). Furthermore, the FRET effect allowed monitoring of DNA-catalyzed site-specific cleavage of the Atto 590-labeled extension of the aptamer. Upon addition of a 10-23 deoxyribozyme with appropriate binding arms, ${ }^{[28]}$ the $\mathrm{DMHBO}^{+}$ emission intensity increased, while Atto 590 intensity decreased concomitantly (Figure $4 \mathrm{~b}$ ). The time-dependent change in the ratio of fluorescence signals $\left(\mathrm{I}_{590} / \mathrm{l}_{620}\right)$ (Figure $4 \mathrm{c}$ ) correlates well with the gel-based analysis (Figure 4d). The polyacrylamide ge was stained with $\mathrm{DMHBO}^{+}$and imaged with green and blue epi illumination (i.e., green: excitation of Atto 590; blue: excitation of $\mathrm{DMHBO}^{+}$). The false-color overlay confirms the presence of both chromophores in the full-length construct and identifies the single-labeled cleavage products (Figure $4 d$, S15). This proof-ofprinciple experiment suggests that the Chili-DMHBO ${ }^{+}$-Atto 590 FRET pair can be used for ratiometric RNA sensors and multicolor fluorescence experiments.
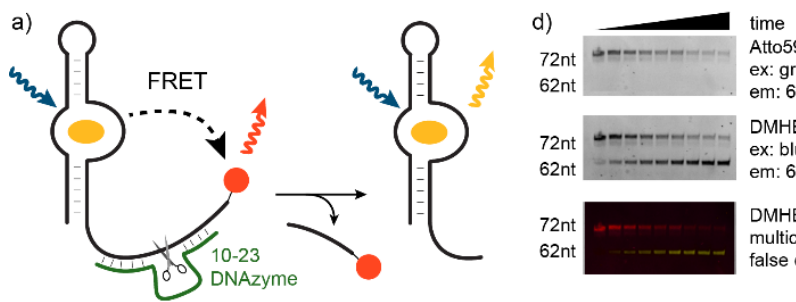
Atto590 ex: green ep $\mathrm{DMHO}^{+}$ ex: blue epi $\mathrm{DMHBO}^{+}$ multichannel false color
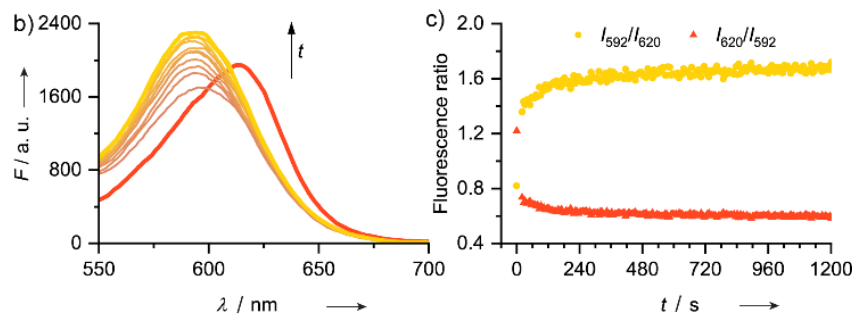

Figure 4. a) A Chili aptamer-based FRET sensor monitors DNA-catalyzed RNA cleavage. Donor: $\mathrm{DMHBO}^{+}$(orange), acceptor: Atto 590 (red). b) Fluorescence emission spectra of Atto 590 -labeled Chili-DMHBO ${ }^{+}$before addition of DNA enzyme (red curve). Upon addition of 10-23 DNA enzyme, spectra were taken every $6 \mathrm{sec}$ (every $20^{\text {th }}$ spectrum shown up to $20 \mathrm{~min}$ ). c) Kinetics of RNA cleavage monitored by a decrease in acceptor emission (red) and an increase in donor emission (orange). d) Denaturing PAGE imaged with green and blue epi illumination after staining with a $1 \mu \mathrm{M}$ solution of $\mathrm{DMHBO}^{+}$. Time points: $0.25,0.5,1,2,5,10,20,40,60 \mathrm{~min}$. Experimental conditions for c) and d): $0.5 \mu \mathrm{M}$ RNA, $5 \mu \mathrm{M}$ DNA enzyme, $40 \mathrm{mM}$ HEPES pH 7.5, $125 \mathrm{mM}$ $\mathrm{KCl}, 5 \mathrm{mM} \mathrm{MgCl}$.
In summary, the Chili RNA aptamer in complex with $\mathrm{DMHBI}^{+}$ and $\mathrm{DMHBO}^{+}$mimics green and red LSS fluorescent proteins. Rational design resulted in the first variants of $\mathrm{HBI}$ aptamer ligands with permanently cationic sidechains. The Chili$\mathrm{DMHBO}^{+}$complex is the longest wavelength-emitting $(592 \mathrm{~nm})$ and tightest binding $\left(12 \mathrm{nM} K_{\mathrm{D}}\right)$ RNA fluoromodule in the growing family of $\mathrm{HBI}$-binding aptamers. In the future, it will be exciting to elucidate the three-dimensional structure of the binding site to comprehend the structural basis of the proton relay in the RNA responsible for the large apparent Stokes shift, and to enable engineering of even brighter LSS aptamer variants for applications in vitro and in cells.

\section{Acknowledgements}

This work was supported by the European Research Council (ERC consolidator grant No. 682586), by the Cluster of Excellence and DFG Research Center Nanoscale Microscopy and Molecular Physiology of the Brain, University of Göttingen, and by the University of Würzburg.

Table 1. Photophysical data of Chili aptamer complexes ${ }^{[a]}$ in comparison to selected fluorescent proteins.

\begin{tabular}{lllll}
\hline Fluorophore & $\mathrm{Ex} \mathrm{Em}^{[\mathrm{b}]}(\mathrm{nm})$ & $\begin{array}{l}\text { Stokes } \\
\text { shift }(\mathrm{nm})\end{array}$ & $\begin{array}{l}\text { Quantum } \\
\text { yield }^{[\mathrm{c}]}\end{array}$ & $K_{\mathrm{D}}(\mu \mathrm{M})$ \\
\hline $\mathrm{DMHBI}$ & $400 / 537$ & 137 & 0.08 & 0.57 \\
$\mathrm{DMHBI}^{+}$ & $413 / 542$ & 129 & 0.40 & 0.063 \\
DMHBI-Imi $^{-}$ & $463 / 545,594^{[\mathrm{d}]}$ & 82,131 & 0.08 & 0.071 \\
DMHBO $^{+}$ & $456 / 592$ & 136 & 0.10 & 0.012 \\
\hline GFP $^{[22]}$ & $475 / 508$ & 33 & 0.79 & - \\
Kaede $^{[21]}$ & $572 / 582$ & 10 & 0.33 & - \\
LSSmOrange $^{[14]}$ & $437 / 572$ & 135 & 0.45 & - \\
LSSmKate2 $^{[15]}$ & $460 / 605$ & 145 & 0.17 & -
\end{tabular}

[a] in $40 \mathrm{mM}$ HEPES pH 7.5, $125 \mathrm{mM} \mathrm{KCl,} 5 \mathrm{mM} \mathrm{MgCl}_{2}$ [b] Excitation and emission maxima. [c] relative quantum yield using Coumarin 153 as standard. [d] Two maxima of the bimodal emission spectrum.

Keywords: RNA aptamer • fluorescence • large Stokes shift • fluorescent protein $\bullet$ fluorescence resonance energy transfer

[1] a) S. Hennig, S. Neubacher, Angew. Chem. Int. Ed. 2018 doi:10.1002/anie.201806482; Angew. Chem. 2018, doi: 10.1002/ange. 201806482; b) S. R. Jaffrey, Adv. Pharmacol. 2018, 82, 187; c) K. Rau, A. Rentmeister, ACS Cent. Sci. 2017, 3, 701; d) S. C. Alexander, N. K. Devaraj, Biochemistry 2017, 56, 5185.

[2] J. R. Babendure, S. R. Adams, R. Y. Tsien, J. Am. Chem. Soc. 2003 , 125, 14716

[3] L. A. Holeman, S. L. Robinson, J. W. Szostak, C. Wilson, Fold. Des. 1998, 3, 423. 
[4] a) M. Sunbul, A. Jäschke, Angew. Chem. Int. Ed. 2013, 52, 13401; Angew. Chem. 2013, 125, 13643; b) M. Sunbul, A. Jäschke, Nucleic Acids Res. 2018, doi: 10.1093/nar/gky543.

[5] M. Gotrik, G. Sekhon, S. Saurabh, M. Nakamoto, M. Eisenstein, H. T. Soh, J. Am. Chem. Soc. 2018, 140, 3583.

[6] X. Tan, T. P. Constantin, K. L. Sloane, A. S. Waggoner, M. P. Bruchez, B. A. Armitage, J. Am. Chem. Soc. 2017, 139, 9001.

[7] A. Autour, S. C Y Jeng, A. D Cawte, A. Abdolahzadeh, A. Galli, S. S. S. Panchapakesan, D. Rueda, M. Ryckelynck, P. J. Unrau, Nat. Commun 2018, 9, 656.

[8] E. V. Dolgosheina, S. C. Y. Jeng, S. S. S. Panchapakesan, R. Cojocaru, P. S. K. Chen, P. D. Wilson, N. Hawkins, P. A. Wiggins, P. J. Unrau, ACS. Chem. Biol. 2014, 9, 2412.

[9] J. S. Paige, K. Y. Wu, S. R. Jaffrey, Science 2011, 333, 642.

[10] G. S. Filonov, J. D. Moon, N. Svensen, S. R. Jaffrey, J. Am. Chem. Soc. 2014, 136, 16299.

[11] W. Song, G. S. Filonov, H. Kim, M. Hirsch, X. Li, J. D. Moon, S. R. Jaffrey, Nat. Chem. Biol. 2017, 13, 1187.

[12] F. Vollmer, W. Rettig, E. Birckner, J. Fluoresc. 1994, 4, 65.

[13] a) O. Zapata-Hommer, O. Griesbeck, BMC biotechnol. 2003, 3, 5; b) H.-W. Ai, K. L. Hazelwood, M. W. Davidson, R. E. Campbell, Nat. Methods 2008, 5, 401.

[14] D. M. Shcherbakova, M. A. Hink, L. Joosen, T. W. J. Gadella, V. V. Verkhusha, J. Am. Chem. Soc. 2012, 134, 7913

[15] K. D. Piatkevich, V. N. Malashkevich, S. C. Almo, V. V. Verkhusha, J. Am. Chem. Soc. 2010, 132, 10762.

[16] Secondary structure was predicted using the Vienna RNA webservices: http://rna.tbi.univie.ac.at/ and compared to the predicted secondary structure reported in Ref. 9.

[17] M. A. Mullen, S. M. Assmann, P. C. Bevilacqua, J. Am. Chem. Soc. 2012, 134, 812.

[18] a) K. D. Warner, L. Sjekloća, W. Song, G. S. Filonov, S. R. Jaffrey, A. R. Ferré-D'Amaré, Nat. Chem. Biol. 2017, 13, 1195; b) H. Huang, N. B.
Suslov, N.-S. Li, S. A. Shelke, M. E. Evans, Y. Koldobskaya, P. A. Rice, J. A. Piccirilli, Nat. Chem. Biol. 2014, 10, 686; c) P. Fernandez-Millan, A. Autour, E. Ennifar, E. Westhof, M. Ryckelynck, RNA 2017, 23, 1788; d) K. D. Warner, M. C. Chen, W. Song, R. L. Strack, A. Thorn, S. R. Jaffrey, A. R. Ferré-D'Amaré, Nat. Struct. Mol. Biol. 2014, 21, 658.

[19] a) R. Trachman, A. Abdolahzadeh, A. Andreoni, R. Cojocaru, J. R. Knutson, M. Ryckelynck, P. J. Unrau, A. Ferré-D'Amaré, Biochem. 2018; b) R. J. Trachman, N. A. Demeshkina, M. W. L. Lau, S. S. S Panchapakesan, S. C. Y. Jeng, P. J. Unrau, A. R. Ferré-D'Amaré, Nat Chem. Biol. 2017, 13, 807

[20] W. Song, R. L. Strack, N. Svensen, S. R. Jaffrey, J. Am. Chem. Soc 2014, 136, 1198.

[21] R. Ando, H. Hama, M. Yamamoto-Hino, H. Mizuno, A. Miyawaki, Proc. Natl. Acad. Sci. U.S.A. 2002, 99, 12651.

[22] a) R. N. Day, M. W. Davidson, Chem. Soc. Rev. 2009, 38, 2887; b) R. M. Wachter, J. L. Watkins, H. Kim, Biochemistry, 2010, 49, 7417.

[23] a) J. M. Lerestif, J. Perrocheau, F. Tonnard, J. P. Bazureau, J. Hamelin, Tetrahedron 1995, 51, 6757; b) A. Baldridge, J. Kowalik, L. Tolbert, Synthesis 2010, 2424.

[24] Scandium triflate was the preferred catalyst compared to more commonly used $\mathrm{ZnCl}_{2}$ in previous reports of analogous reactions: W.-T. Chuang, B.-S. Chen, K.-Y. Chen, C.-C. Hsieh, P.-T. Chou, Chem. Commun. 2009, 6982.

[25] G. Feng, C. Luo, H. Yi, L. Yuan, B. Lin, X. Luo, X. Hu, H. Wang, C. Lei, Z. Nie et al., Nucleic Acids Res. 2017, 45, 10380.

[26] S. Bose, S. Chakrabarty, D. Ghosh, J. Phys. Chem. B 2017, 121, 4790.

[27] A 20-nt 3'-amino-modified RNA was labeled with Atto 590-NHS ester and ligated to the Chili RNA with T4 RNA ligase. Experimental details are given in the Supporting information

[28] a) S. W. Santoro, G.F. Joyce, Proc. Natl. Acad. Sci. USA, 1997, 94, 4262; b) S.K. Silverman, Nucleic Acids Res. 2005, 33, 6151. 


\section{Entry for the Table of Contents}

\section{COMMUNICATION}

"My shadow side so amplified." (Red Hot Chili Peppers)

A large Stokes shift fluorogenactivating RNA aptamer (LSS FLAP) named "Chili" strongly amplifies green or red fluorescence emission of otherwise non-fluorescent, tightly bound cationic ligands upon excitation with blue light. Chili allows visualization of RNA and can be used in FRET sensors.

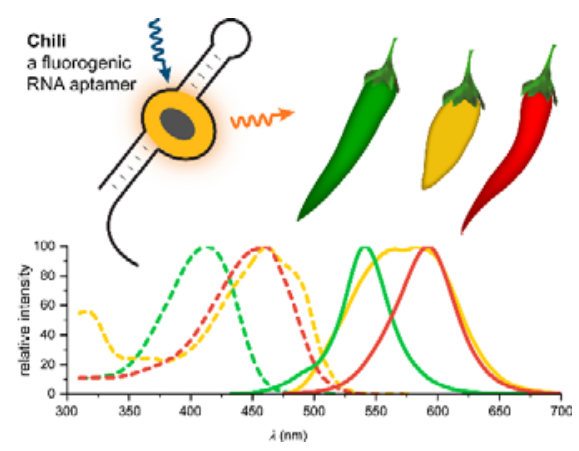

C. Steinmetzger, N. Palanisamy, K.R. Gore, C. Höbartner*

Page No. - Page No.

A multicolor large Stokes shift fluorogen-activating RNA aptamer with cationic chromophores 